\title{
Luminal Gastrointestinal Surgery: What Is Round the Corner?
}

\author{
Sharad Karandikar • Sudeep Shah • \\ Chiranjiva Khandelwal
}

Received: 27 October 2014 / Accepted: 28 November 2014 / Published online: 23 December 2014

(C) Association of Surgeons of India 2014

In the kaleidoscope of general surgery, upper and lower gastrointestinal surgery, hepatopancreatobiliary surgery and emergency surgery take different forms depending on which continent, country, city the practice is based and varies from individual to individual.

"No man is a pure specialist without being in the strict sense an idiot." George Bernard Shaw (1856-1950)

Gastrointestinal surgeons all over the world are "generalists" in emergency surgical practice and "specialists" in elective work. From a patient perspective, safe cure from their symptom with minimum morbidity and improved quality of life, with the freedom to make an informed choice, is paramount. Following on their success after the hepatobiliary and transplant issue, Indian Journal of Surgery has commissioned a special issue exploring some of the emerging challenges in management of the wide spectrum of luminal gastrointestinal diseases from reflux disease to cancer.

Abdominal surgical emergencies remain the most challenging part of surgical practice as it is associated with the highest mortality. Advances in imaging and minimally invasive therapies and their access to patients and surgeons are ever improving. Better understanding of the disease process and range of interventions including wider range of antibiotics for acute

S. Karandikar

Birmingham Heartlands Hospital, Birmingham, UK

e-mail: Sharad.karandikar@heartofengland.com

S. Shah

Division of GI Surgery, PD Hinduja Hospital, Mumbai 400016, India

e-mail: shahsudeep@hotmail.com

C. Khandelwal $(\bowtie)$

G.I. Surgery, Indira Gandhi Institute of Medical Sciences, Patna 800014, India

e-mail: khandelwal3250@gmail.com inflammatory pathology in the abdomen (including appendicitis, cholecystitis and diverticulitis) has allowed tailoring intervention for appropriate patients. Hartley et al. [1] illustrate this principle in the management of diverticulitis. Laparoscopy has established itself a place in the management in appendicitis and continues to push the limits as Khetan et al. [2] share the evidence supporting even considering it for intestinal obstruction. Laparoscopic surgery is now challenging the "gold standard" of open surgery in cancer surgery; Shrikande et al. [3] try to make a case for this in appropriately selected patients.

Displaced viscera due to failure of the abdominal defences (hiatus hernia and full-thickness rectal prolapse) has offered surgeons challenges over decades. In both these conditions, operation is for improving quality of life and choice of wide range of surgical procedures poses surgeons with peculiar difficulties. Khaitan et al. [4] share their interpretation of the current literature. Prospective randomised evidence (PROSPER trail) for rectal prolapse confirms difficulties in treatment choices and outcomes. McArthur et al. [5] interpret the existing evidence for laparoscopic options in the backdrop of the PROSPER trial. Upper gastrointestinal surgery has a new kid on the block of bariatric surgery; Sarela et al. [6] give an insight into the debates currently in this emerging field.

"While there are several chronic diseases more destructive to life than cancer, none is more feared." Charles Mayo

Gastrointestinal cancer continues to dominate the patients, surgeons and researchers' interest in the twenty-first century. The proximal- and distal-most parts of the gastrointestinal tract cancers due to their anatomy and pathophysiology peculiarities have moved into specialist territory sooner. Pramesh et al. [10] revisit the debates in the current management of 
oesophageal cancers. The more we know, the less we understand; rectal cancer epitomises this in the GI tract. Evan et al. [7] share their vision in terms of the mist of evidence and how it can be translated into individualising it to patients.

Arguably, fistula in ano surgery is a discriminator between a general surgeon and a proctologist. Sheikh et al. [8] share the current tools in assessment and identification of complex diseases and available therapeutic choices for successful outcomes in this benign but difficult disease.

"Grant me an opportunity to improve and extend my training, since there is no limit to knowledge. Help me to correct and supplement my educations defects as the scope of science and its horizon widen day by day." Moses ben Maimon (Maimonides) 1135-1204

Technology provides new generation of surgeons with additional learning tools like simulation training which are becoming popular. Aryal et al. [9] share the concept of "Virtual Learning Environment" and how it could become a part of surgical training in the years to come.

\section{References}

1. Welbourn H, Hartley JE (2014) Management of acute diverticulitis and its complications. Indian J Surg. doi:10.1007/s12262-014-1086-6

2. Gupta A, Khan M, Harikrishnan A, Khetan N (2014) Laparoscopic surgery in luminal gastrointestinal emergencies - a review of current status. Indian J Surg. doi:10.1007/s12262-014-1081-y

3. Shrikhande SV, Gaikwad V, Desouza A, Goel M (2014) Is laparoscopic surgery the standard of care for GI luminal cancer? Indian J Surg. doi:10.1007/s12262-014-1126-2

4. Singhal V, Khaitan L (2014) Gastro-esophageal reflux disease: diagnosis and patient selection. Indian J Surg. doi:10.1007/s12262-014-1090-x

5. Shastri-Hurst N, McArthur DR (2014) Laparoscopic rectopexy for rectal prolapse: will it be the gold standard? Indian J Surg. doi:10. 1007/s12262-014-1088-4

6. Sarela AI (2014) Informed decision-making for bariatric surgery: benefits, risks, uncertainties \& choices. Indian J Surg. doi:10.1007/ s12262-014-1103-9

7. Rawat N, Evans MD (2014) Paradigm shift in rectal cancer. Indian J Surg. doi:10.1007/s12262-014-1089-3

8. Sheikh P, Baakza A (2014) Management of fistula in ano-the current evidence. Indian J Surg. doi:10.1007/s12262-014-1150-2

9. Aryal K, Pereira J (2014) E learning in surgery. Indian J Surg. doi:10. 1007/s12262-014-1092-8 